\title{
No effect of vitamin D supplementation on circulating concentrations of matrix metalloproteinase-9 (MMP-9) and tissue inhibitor of metalloproteinases-1 (TIMP-1) in adults aged 20-40 and $\geq 64$ years
}

\author{
A. Lucey $^{1}$, T. Hill ${ }^{1}$, S. Muldowney ${ }^{1}$, K. Seamans ${ }^{1}$ J. M. W. Wallace ${ }^{3}$, G Horigan $^{3}$, M. S. Barnes ${ }^{3}$, \\ K. D. Cashman ${ }^{1,2}$ and M. Kiely ${ }^{1}$ \\ ${ }^{1}$ Department of Food \& Nutritional Sciences and ${ }^{2}$ Department of Medicine, University College Cork, Cork, Republic of \\ Ireland and ${ }^{3}$ Northern Ireland Centre for Food and Health, University of Ulster, Coleraine BT52 1SA, UK
}

Tissue matrix metalloproteinases participate in extracellular matrix remodelling and degradation. Increased expression of MMP-9 and its inhibitor TIMP-1 are linked to unfavourable cardiovascular conditions, including inflammatory damage leading to increased plaque instability ${ }^{(1)}$. Vitamin D insufficiency is associated with higher MMP-9 concentrations, while vitamin D supplementation has been shown to decrease circulating MMP-9 and TIMP-1 concentrations in vitamin D-deficient adults ${ }^{(2)}$.

The effect of vitamin D supplementation $(0,5,10$ and $15 \mu \mathrm{g}$ cholecalciferol/d) on MMP-9 and TIMP-1 concentrations was investigated in two randomised placebo-controlled double-blind 22-week intervention studies in men and women aged 20-40 years ( $n$ 215; during winter 2006- $7^{(3)}$ ) and $\geq 64$ years ( $n$ 215; during winter 2007- $8^{(4)}$ ) from Cork and Coleraine. Fasting serum levels of MMP-9, TIMP-1 and 25-hydroxyvitamin D (25(OH)D) were measured by ELISA at baseline and end point.

\begin{tabular}{|c|c|c|c|c|c|c|c|c|c|c|c|c|c|c|c|c|c|}
\hline & \multicolumn{16}{|c|}{ Treatment group ( $\mu$ g cholecalciferol/d) } & \multirow[b]{4}{*}{$P$} \\
\hline & \multicolumn{4}{|c|}{ Placebo } & \multicolumn{4}{|c|}{5} & \multicolumn{4}{|c|}{10} & \multicolumn{4}{|c|}{15} & \\
\hline & \multicolumn{2}{|c|}{$\mathrm{BL}$} & \multicolumn{2}{|c|}{ EP } & \multicolumn{2}{|c|}{$\mathrm{BL}$} & \multicolumn{2}{|c|}{ EP } & \multicolumn{2}{|c|}{$\mathrm{BL}$} & \multicolumn{2}{|c|}{$\mathrm{EP}$} & \multicolumn{2}{|c|}{$\mathrm{BL}$} & \multicolumn{2}{|c|}{$\mathrm{EP}$} & \\
\hline & Mean & $\overline{\mathrm{SD}}$ & Mean & $\mathrm{SD}$ & Mean & SD & Mean & SD & Mean & $\mathrm{SD}$ & Mean & $\mathrm{SD}$ & Mean & $\mathrm{SD}$ & Mean & SD & \\
\hline $20-40$ years & & \multicolumn{3}{|c|}{$(n 56)$} & \multicolumn{4}{|c|}{$(n 50)$} & \multicolumn{4}{|c|}{$(n 57)$} & \multicolumn{4}{|c|}{$(n$ 52) } & \\
\hline MMP-9 (ng/ml) & 237 & 175 & 187 & 147 & 222 & 177 & 183 & 150 & 242 & 200 & 197 & 166 & 238 & 17 & 210 & 185 & 0.381 \\
\hline TIMP-1 (ng/ml) & 158 & 34 & 156 & 38 & 167 & 37 & 159 & 41 & 161 & 39 & 152 & 37 & 171 & 39 & 155 & 39 & 0.441 \\
\hline $25(\mathrm{OH}) \mathrm{D}(\mathrm{nmol} / \mathrm{l})$ & 76.8 & 33 & $41.8^{\mathrm{a}}$ & 18 & 71.3 & 27 & $53.4^{\mathrm{b}}$ & 15 & 77.6 & 33 & $62.1^{\mathrm{c}}$ & 22 & 79.7 & 30 & $72.4^{\mathrm{d}}$ & 21 & $<0.001^{(3)}$ \\
\hline$\geq 64$ years & \multicolumn{4}{|c|}{$(n 56)$} & \multicolumn{4}{|c|}{$(n 51)$} & \multicolumn{4}{|c|}{$(n$ 57) } & \multicolumn{4}{|c|}{$(n 51)$} & \\
\hline MMP-9 (ng/ml) & 205 & 189 & 251 & 233 & 188 & 192 & 211 & 203 & 197 & 206 & 196 & 187 & 180 & 172 & 229 & 228 & 0.603 \\
\hline TIMP-1 (ng/ml) & 178 & 42 & 186 & 43 & 186 & 49 & 188 & 54 & 182 & 48 & 175 & 42 & 187 & 37 & 185 & 41 & 0.815 \\
\hline $25(\mathrm{OH}) \mathrm{D}(\mathrm{nmol} / \mathrm{l})$ & 58.9 & 23 & $43.1^{\mathrm{a}}$ & 17 & 57.9 & 23 & $58^{\mathrm{b}}$ & 16 & 59.2 & 26 & $70.6^{\mathrm{c}}$ & 18 & 53.73 & 18 & $76.2^{\mathrm{c}}$ & 21 & $<0.001^{(4)}$ \\
\hline
\end{tabular}

$\mathrm{BL}$, baseline; EP, end point (EP). Means in a row with unlike superscript letters were significantly different $(P<0.001)$

ANOVA showed no baseline differences in the circulating concentrations of MMP-9, TIMP-1 or 25(OH)D between the four treatment groups. Baseline MMP-9 and TIMP-1 concentrations were significantly higher in adults aged 20-40 years $(P<0.001)$ and adults aged $\geq 64$ years $(P<0.01)$ in Coleraine than in Cork. Linear regression analysis showed study centre to be the main predictor of MMP-9 (adjusted $R^{2} 0.474 ; P<0.001$ ) and TIMP-1 (adjusted $R^{2} 0.326 ; P<0.001$ ) concentrations in adults aged 20-40 years. In both age-groups pre- and post-intervention 25(OH)D concentrations were not associated with levels of MMP-9, TIMP-1 or MMP-9:TIMP-1. In both agegroups repeated measures analysis revealed no significant effect of the intervention on MMP-9 and TIMP-1 concentrations across the four groups, adjusting for centre, age, gender and BMI.

In conclusion, vitamin D supplementation had no effect on circulating MMP-9 and TIMP-1 concentrations in apparently-healthy adults aged $20-40$ and $\geq 64$ years.

We wish to acknowledge the UK Food Standards Agency and the Irish Department of Agriculture, Food \& Fisheries through the Food Institutional Research Measure for their support.

1. Janssens S \& Lijnen HR (2006) Cardiovasc Res 69, 585-594

2. Timms PM, Mannan N, Hitman GA et al. (2002) Q J Med 95, 787-796.

3. Cashman KD, Hill TR, Lucey AJ et al. (2008) Am J Clin Nutr 88, 1535-1542.

4. Cashman KD, Wallace JMW, Horigan G et al. (2009) Am J Clin Nutr 89, 1-9. 